The philosopher Immanuel Kant said, "Two things fill the mind with ever new and increasing admiration and awe, the oftener and the more steadily we reflect on them: the starry heavens above and the moral law within" (Kant, 2004 [1788]: 170). As a child I had fancied becoming an astronomer. Alas, those hopes were dashed when I learned that to become an astronomer, one must enjoy mathematics. So I turned instead (many years later) to the moral law within - the self - and in particular to autonomy and authenticity, being among the central concepts in bioethics and philosophy of medicine. Such concepts have, of course, been explored by philosophers for thousands of years, but I sought to understand what these concepts mean to people in real situations when those elements of the self are most acutely challenged.

As so much of our ideas concerning the self draw on our experiences and intuitions of what it is to have a self and to feel true to it, it seemed that while armchair theorising certainly has its function, even more can be learned from empirical studies. Moreover, although classified as a medical condition, depression also contains very personal and social aspects which are integral to the experience. Illuminating this human element of depression rather than just the clinical or the purely theoretical advances our knowledge of it in a way that may not be possible if scholarship is focused solely on theory (which may or may not have any bearing on reality), or on clinical aspects, or indeed solely on the experiences of individuals without relating the significance of those experiences to current theory. While physical illnesses do impact on the self in various ways, mental illnesses, by affecting one's thoughts, emotions and behaviours, directly impact the various elements which we take to form the self. Depression, being apparently the most common mental illness, seemed to be the perfect candidate for such an exploration into the self.

There is barely a person who either has not been directly touched by depression or does not know someone who has. Now considered the leading cause of disability worldwide and a major contributor to the 
global disease burden, depression is said to affect staggering numbers of people - an estimated 300 million around the world according to the World Health Organization (2017). There is also a large literature on it to match - everything from scientific studies searching for causes and cures to autobiographical accounts written by those who continue to struggle with it or who have survived. This book is somewhat different in that it focuses on specific aspects of the self in depression.

Aside from the suffering inherent in depression, there is another difficulty which individuals who have experienced depression often face. Annette articulates this difficulty succinctly:

Annette: When you've done things or said things or thought things or behaved in an inappropriate way, you think, "Are people gonna see this as part of my personality? Is this part of who I am? Or you know, is this just how I behaved because my chemicals weren't right?"

As Annette describes, it is a question of where to assign responsibility for emotions, thoughts and behaviours which are normally considered to be part of one's sense of self but which, with a diagnosis of depression and subsequent treatment, now come under question. This problematises the way patients and the general public think about the self in relation to depression.

Prior research has presented accounts of patients on antidepressants who felt as though they went from an "old" to a "new" self, or had found their authentic self (Karp, 2006; Kramer, 1993). I sought to learn why some people feel like their true self while on antidepressants whereas others feel the opposite, why some describe depression as an illness ready to attack, while others describe it as a part of their self. I wondered if perhaps their views related in some way to their experiences of depression and treatment. Essentially, I sought to go beyond an examination of the effects of depression and psychiatric medication to see if individuals' reflections on their experiences of depression and treatment shed any light on our concepts of the self.

The women whose experiences I draw on in this book participated in a study conducted in England. The study aimed to investigate if and how aspects of women's experiences of depression, diagnosis and treatment relate to their perceptions of the self in depression and recovery. I included 37 women with different diagnoses and treatments in order to explore a broad range of experiences. As such, there were no limits placed on type, recurrence, duration or nature of depression (although minor depression was excluded) or type of treatment. In order to qualify for inclusion in the study, interviewees had to be female, be between the ages of 18 and 65, have been diagnosed with depression, have received 
treatment for it, and be feeling better (according to both the individual's judgement and her Beck score at the time of interview). ${ }^{1}$ I included the latter condition in order to recruit individuals in a relatively stable condition so that they could reflect on their experiences of depression, treatment and recovery and to minimise the potential for emotional distress during the interview.

Seeking to focus on experiential aspects of depression rather than on the already well-studied gender and socio-economic aspects of depression, I realised this could best be done if I focused on one gender and on individuals with similar educational and socio-economic backgrounds. A number of studies have provided evidence of gender differences related to depression, citing both social and biological factors which could influence both the development of depression (e.g. Goodwin \& Gotlib, 2004; Kessler, 2003; Kueher, 2003; Piccinelli \& Wilkinson, 2000) and its course and responses to certain treatments (Barnes \& Mitchell, 2005; Burt \& Rasgon, 2004; Freeman \& Gelenberg, 2005; Leibenluft, 1996, 1997). Studies have also documented female versus male narrative differences (e.g. Buckner \& Fivush, 1998; Thompson, 1998), and there have already been several analyses of gender differences in depression experiences (Danielsson et al., 2009; Danielsson \& Johansson, 2005; Danielsson et al., 2010; Emslie et al., 2007; Emslie et al., 2006; O'Brien et al., 2005). Setting aside gender differences thus allowed me to narrow the focus of my investigation onto other factors which may account for differences in individuals' accounts. This book is therefore not an investigation of the gender-related social issues surrounding the causes, diagnosis and treatment of women's depression. Rather, it is an investigation of how experiences of depression impact on certain aspects of the self.

I chose to interview women rather than men, firstly, because rates of depression are widely reported to be twice as high for women, as measured by both community surveys and diagnosed and treated cases (Angst $e t$ al., 2002; Bromet et al., 2011; Steffens et al., 2000), which meant it would likely be easier to find volunteers among the female population than among the male population. Secondly, I was aware that being of the same gender may enable the interviewees to identify with me and feel comfortable sharing their experiences. As Benney et al. (2003: 46) contend, "the least inhibited communication seems to take place between young people of the same sex". Being in my mid-to-late twenties at the time of the interviews, I also fit with the age range of most of the interviewees.

Given my research focus on different experiences of depression and treatment, it made sense to try to limit variation in other factors as much as possible. For this reason, I initially set out to select participants from one location. When recruitment within one city alone 
proved difficult and alternative means of recruitment other than through a psychiatrist were exhausted, I looked into additional locations from which to recruit. The two main study sites are unique due to their disproportionately educated, middle-class populations compared to many other parts of England. The sample of women who participated in the study reflects this and most were educated to university level. The sample was also predominantly white and fairly young - the average age was 32.8 years - and the largest age group by far was women in their twenties, with 18 of the 37 women in this age group. Although the characteristics of the sample limit the generalisability of the study's findings, it allowed me to focus on interpretive factors and the variety of experiences of depression, diagnosis and treatment rather than on the variety of experiences across gender, age, social class and ethnic groups which have been investigated in many other depression studies (e.g. Estroff et al., 1991; Pilgrim \& Rogers, 1993).

My focus on finding meanings within individuals' perceptions of their experience is patently a phenomenological investigation (Van Manen, 1984). As such, a phenomenological analysis was the most obvious analytic method for me to employ. Both descriptive and interpretative phenomenological analyses aim to capture the "quality and texture of the individual experience" (Willig, 2008: 57). Yet interpretative phenomenology recognises that such an analysis is always the researcher's interpretation of that experience. Moreover, rather than aiming to elucidate the general structure of how a particular phenomenon is experienced, as is the aim in descriptive phenomenology (Giorgi \& Giorgi, 2008), my aim was more closely aligned with those of the interpretative branch in both its focus on perceptions (Kvale, 1996) and its attempt to interpretatively engage with material (whether they be texts or transcripts) to unravel the meanings within them (Smith, 1997). Its aim is to try to see the world through the participant's eyes and to take that insider's view as much as possible (Crossley, 2000). It does so by "allowing participants to tell their story, in their own words, about the topic under investigation" (Smith et al., 1997: 68). As a result, interpretative phenomenology was a major influence on the analytic framework.

\section{Defining Depression}

Many people who have experienced depression say it is indescribable. My own perspective is as someone who has learnt about depression through reading studies and autobiographies, as well as speaking to others about their experience of depression. As I do not have a first-hand account of what depression is like, I speak as a bystander rather than as a survivor. My view, therefore, is as someone from the outside rather than within. 
In addition to the experience of depression itself, the issue of the label the individual acquires may also have a significant influence on how the individual perceives depression in relation to herself. It is for this reason that I decided to use the clinician's diagnosis of depression, regardless of how accurate the diagnosis may be (or indeed, if a diagnosis is appropriate at all), as the criterion for inclusion rather than using other criteria such as the Beck depression scale or applying the diagnostic criteria in the Diagnostic and Statistical Manual of Mental Disorders (DSM). This allowed me to investigate the influence that the diagnosis itself has on how the individual conceptualises what it is she is suffering from and its role in her life and sense of self. As a result, rather than relying on so-called objective criteria for depression (which has the added risk of reifying the category), I embrace the subjectivity of the clinician making the diagnosis as a relevant and important part of an examination into the self and depression.

\section{Defining the Self}

In order to conduct an investigation into the self in depression, one must first be clear on what is meant by "the self". To this end, this section gives an overview (which is by no means exhaustive) of different definitions of the self to give the reader an impression of the landscape, before identifying which definition is given the most support by my empirical investigation. The term "self" is a concept which, as Epstein aptly describes, is "a slippery concept whose adequate definition is irritatingly elusive" (1973: 404), resulting in numerous definitions. Merriam-Webster's Online Dictionary defines the self as:

$1 \mathbf{a}$ : the entire person of an individual

$\mathbf{b}$ : the realization or embodiment of an abstraction

2 a(1) : an individual's typical character or behavior "her true self was revealed"

(2) : an individual's temporary behavior or character "his better self"

b : a person in prime condition "feel like my old self today"

3 : the union of elements (as body, emotions, thoughts, and sensations) that constitute the individuality and identity of a person. (Merriam-Webster's Online Dictionary, 2010)

The New Shorter Oxford English Dictionary includes the following in its definition of the self:

A person's or thing's individuality or essence at a particular time or in a particular aspect or relation; a person's nature, character, or (occas.) physical constitution or appearance, considered as different at different times ...

True or intrinsic identity; personal identity, ego; a person as the object of introspection or reflexive action. (Brown, 1993: 2763) 
These dictionary definitions give an indication of the variety of conceptions of the self. Within the literature, there is first a distinction between the self at the individual level and the self at the societal level (Ashmore \& Jussim, 1997). At the individual level, authors usually distinguish between the self as subject (or agent) and the self as object (or observed entity) (Ashmore \& Jussim, 1997). William James first referred to the distinction as "self as knower" and "self as known", or the "I" and "Me" or "subject" and "object" distinction, respectively (James, 1890). Several authors have attempted to identify the elements of the self as subject and object (e.g. Damon \& Hart, 1982, 1986; Harter, 1983).

A second distinction within the concept of the self at the individual level is that between self-conception and self-evaluation: that is, between the evaluative and descriptive parts of the self (Beane \& Lipka, 1980; Blyth \& Traeger, 1983; Greenwald et al., 1988; Hogg \& Cooper, 2003). A third distinction centres on the question of whether the self is stable or constantly changing. Some believe the self reflects time-specific and contextual factors - that it is socially constructed (Gergen, 1977; Linville \& Carlston, 1994) - whereas others believe there is an underlying core self (Epstein, 1980; Markus \& Kunda, 1986; Markus \& Nurius, 1986).

On a societal level, first is the cultural notion of what defines a person, or "the shared conception of the person or individual" (Spiro, 1993: 114). Second is the cultural notion of what defines a self, or "the cultural conception of some psychic entity or structure within the person, variously described as 'pure ego,' 'transcendental ego,' 'soul,' and the like" (Spiro, 1993: 114). Third, and on a more practical level, there are "the bounds on the nature, content, and structure of individual persons and selves set by the current political, legal, economic, and informational institutions, as well as prevailing cultural values and accepted interpersonal processes" (Ashmore \& Jussim, 1997: 8). Fourth, on a more specific level, there are the self-concepts of individuals within a given culture (Ashmore \& Jussim, 1997). These four levels of the self, which of course are not discrete but overlap with each other, can be compared across societies, cultures and time. For instance, researchers interested in concepts of the self across different cultures usually study a society's conception of personhood (Spiro, 1993: 117).

Concepts of the self can also be divided along disciplinary lines. In philosophy, "the self" (or identity) is considered to be the locus of agency, responsibility and personal identity (Mackenzie \& Atkins, 2008), but there is disagreement as to whether it is what constitutes an individual at a point in time, or at different points in time (Blackburn, 2008). Philosophers concerned with the self at one point in time contend that it is possible for more than one person to share the same body, so they ask 
what it is that gives us the impression that we are only one person (Locke, 1959). Philosophers concerned with what constitutes the self at different points in time note that we all change throughout our lives. We are different in some ways from the way we were, say, ten years ago, and may change again in another ten years. This raises the question of what evidence there is to say that I am the same person now as I was ten years ago. It is possible for someone else to have taken over my body in that time (Blackburn, 2008). Alternatively, my psychology may have changed drastically after an event, which problematises the extent to which I can be deemed to be the same person simply because my body has survived (Blackburn, 2008). Some of those I interviewed struggled with a similar issue, speculating as to whether depression had changed their self or whether they would have changed anyway with the passage of time, particularly when their history with depression spanned several years or even decades. Some felt so different during an episode of depression or mania, or when on medication, that they felt like a very different self, or not their true self.

Aristotle defined the self (which he termed the soul) as the essence of a human in the sense that it is the activity of the body. Specifically, it is the potential for rational activity that is the essence of a human self (Aristotle, 2001). In contrast, Ibn Sina (Avicenna) argued that the self is a substance independent of physical components. He postulated that if one was suspended in the air without any sensation whatsoever of external surroundings or even with one's own body, one would still have consciousness of one's self. As a result, the self is not dependent on anything physical (Goodman, 1992).

David Hume believed that the self does not have a constant, underlying essence, but is constituted of a variety of different, fluid elements which are interrelated. At any time, we are merely a bundle of perceptions that do not belong to anything. "We are never intimately conscious of anything but a particular perception; man is a bundle or collection of different perceptions which succeed one another with an inconceivable rapidity and are in perpetual flux and movement" (Hume, 2007 [1739]: I, IV, vi). Daniel Dennett argues that the self is not a physical substance but a "convenient fiction" like the centre of gravity. Especially when making sense of the world, the self is a convenient concept to invoke. But the fictional character at the centre of these stories is the self (Dennett, 1992). While this book cannot speak to the metaphysical question of "What is the self?" there are, nevertheless, instances when one's answer to this question influences one's ideas surrounding the concept of the self in depression. For instance, a dualist view of the self which conceives it as independent of the body may lend itself more easily to a biomedical 
view of depression as an illness, caused by a neurochemical imbalance, that is distinct from the self. As shown in Chapter 2, most of those who professed to hold such a view struggled to maintain it. Yet the view still exerts its influence on many, whether or not they actually espouse it.

Psychology focuses on the self at the level of the individual. William James, whom many consider to be the father of psychological and sociological analyses of the self (Ashmore \& Jussim, 1997), was followed by Calkins (1900), who advocated for psychology to become a science of selves. Apart from those who emphasised the function of the ego and the id, psychoanalysts following Calkins largely ignored the study of the self for some time (Scheibe, 1985). The 1940s saw a revival of the study of the self when researchers created a self-report measure of self-esteem, and for the following 30 years this became a major arena in psychological studies of the self (Wylie, 1974). Apart from self-esteem, psychology has also become concerned with aspects such as self-states, self-motives, selfawareness, self-knowledge and self-image (Leary \& Tangney, 2003). Psychological theories of self-discrepancy (Higgins, 1987), self-concept (Rosenberg, 1979) and psychotherapeutical consistency (Grawe, 2004) have also investigated the self.

As Ashmore and Jussim (1997) note, the terms "self" and "identity" within psychology overlap with concepts such as "personality" and "memory" which help to define the attributes and idiosyncrasies that make us who we are. For example, research into autobiographical memory has found certain personality features to be strongly connected to memories (McAdams, 1982, 1985; McAdams et al., 1997; Woike, 1995; Woike et al., 1999). Memories can also contribute to self-schemas (Habermas \& Bluck, 2000; Markus, 1977), and certain memories have been called "self-defining" because they become crucial to the development of goals and mental well-being (Singer \& Salovey, 1993). Memories can also contribute to generation identity (Conway, 1997; Conway \& Haque, 1999) and the formation of a stable self-system (the individual's set of self-perceptions) (Beike \& Landoll, 2000; Conway \& Rubin, 1993; Conway \& Tacchi, 1996; Fitzgerald, 1988, 1996). Conway and Pleydell-Pearce also note connections between memories and the working self, goals, emotion and the self-memory system (Conway \& Pleydell-Pearce, 2000). On the other hand, Erikson distinguished between "the self" as a continuous ego identity, "personal identity" as the set of idiosyncrasies that make each person unique and "social identity" or "cultural identity" as the social roles a person plays (Wallerstein \& Goldberger, 1998). According to Harré, the self is "that inner unity to which all personal experience belongs as attributes of a subject" (1987: 42). 
Although the women I spoke with recalled memories associated with their depression during the course of their interviews, it is important to distinguish between these accounts and those of autobiographical memory and the self in that; rather than investigating the nature of their memories and their specific connections to aspects of the self, I examine whether simply perceiving the presence or absence of memories of events or circumstances in close connection with depressive episodes is meaningful to how one conceives one's future self. Allowance for the possibility of the absence of memory being meaningful in itself is thus a key methodological difference between this investigation and those concerned with autobiographical memory.

In anthropology, Hallowell is the main figure recognised as having begun an anthropological study of the self (Ashmore \& Jussim, 1997). ${ }^{2}$ Hallowell (1955) believed that the self is "culturally constituted", meaning that the individual's sense of self and the way she evaluates her self is a product of her culture. Potter and Wetherell (1987) argue that cultural discourses frame the way we talk about ourselves, and in turn our experience of personhood. As discourses differ between cultures, individuals in different cultures would experience being a "self" differently. In a similar vein, Harré contends that the structure of our language frames our beliefs about the self (Harré, 1985, 1989). ${ }^{3}$ In contrast, Spiro (1993) argues that Hallowell believed in certain universal ideas about the self commonly held among cultures, and Baumeister (1987) builds on this notion by suggesting what those ideas may be. Burr (2003), on the other hand, believes it is the concept of self that is universal. These anthropological concerns should be kept in mind given that the views of the self among those I interviewed may differ with the views of the self expressed in other cultures.

The above discussion provides a rough "lay of the land" on which to appreciate where this investigation is situated within the broader picture. The definition of the self used in this book overlaps with its meaning within a particular branch of psychology (as presented in the next section). ${ }^{4} \mathrm{I}$ am particularly concerned with how the individual incorporates (or doesn't incorporate) depression into her concept of self (i.e. what attributes do and do not constitute her true self/authenticity in her eyes), how she compares her present self with her past self and future self (which can be viewed as self-states), her medicated self with her non-medicated self (also self-states) and what she considers to be her true self and why (again, attributes of the self). However, the way I investigate the self uses tools found in sociological studies of the self in illness.

Although I do not focus specifically on self-esteem, the issue arises indirectly in many interviews, especially with regard to the individual's 
faith in her ability to manage or overcome depression, and her view of herself in general as both a product of and causal factor in her development of depression. The individual's beliefs regarding her true self also tie in with her self-esteem. Susan Harter conducted a study which showed that the repeated performance of a false self lowers one's self-esteem, which in turn contributes to depression (Harter, 1997). However, the relationship could also perhaps be inverted, with depression and low selfesteem causing the individual to believe that her true self is not good enough and must be masked by a false self. Although I do not investigate this relationship per se, I explore issues surrounding authenticity and the selves which those interviewed choose to describe and present.

Deci and Ryan's (1991, 1995) self-determination theory postulates that competence, relatedness and autonomy are essential to the self. Competence is conceived as mastering particular skills and considering oneself capable and effective. Relatedness is the need to interact and connect with others, and autonomy is defined here as a need to act in ways that derive from the self. As Baumeister (1999: 13) explains:

Failing to fulfil the need for competence leaves the self feeling helpless, useless, and incompetent, whereas failing to fulfil the need for autonomy leaves the self feeling that its actions are dictated by external forces.

Kasser and Ryan contend that happiness and well-being depend on these "intrinsic needs". Their research found that people who focus on gaining "extrinsic goals" such as money and fame have a lower well-being (as measured by factors such as levels of anxiety, depression, selfactualisation and vitality) and that true self-esteem is based on a quest for competence, autonomy and meaningful relationships (Kasser \& Ryan, 1993, 1996). This book examines the relationship between the self, control and well-being (specifically depression) from the opposite perspective. Rather than examining the self and control and measuring what effects different states of control have on well-being, this book focuses on one state of well-being - depression - and examines the relationship between the self and control vis-à-vis that state.

\section{The Self in Existential Psychotherapy}

The concept of the self that is most relevant to this investigation is that found within a particular strand of existential psychotherapy. Existential psychotherapy is based on the belief that the root of most psychological problems is when the individual comes face-to-face with the "givens of existence", resulting in an inner conflict (Yalom, 1980). According to Yalom, these givens (or ultimate concerns) are (1) death, (2) isolation, (3) 
meaninglessness and (4) freedom. I have changed the order in which they are normally presented in order to provide a brief overview of the first three themes before discussing the theme that arose repeatedly in the interviews - freedom.

\section{(1) Death}

According to Becker (1973), to be constantly aware of death would be too overwhelming, so one must be aware of death yet not be overpowered by this awareness. There are several mechanisms with which people deal with the reality of death. Becker (1973) and Yalom (1980) use the concept of the hero, in which people seek to become a hero (i.e. doing something that they think is valuable and will be remembered) in the hope that by doing so their memory will live on, and symbolically they will never die. Paul Tillich (1952) contends that facing death should mean not only facing the end of physical life but also the possibility of nonbeing. According to Tillich, nonbeing is another way to avoid death, as some people believe that if they do not really live, they will not really die. Avoiding relationships (interpreted as being) in order to avoid the pain of rejection (nonbeing) is one example of this.

Death also symbolises that over which humans have no knowledge and no control. Kierkegaard's view on this subject is taken from the biblical story of the Fall of Adam and Eve from the Garden of Eden. Here, humans emerge from instinctive animal action into a consciousness of our individuality, but simultaneously a consciousness of our death and decay. According to Kierkegaard, it is our awareness of our own death which is our greatest anxiety (Kierkegaard et al., 1980).

\section{(2) Isolation}

In existential therapy, isolation does not refer to interpersonal isolation (isolation from others) or intrapersonal isolation (isolation from parts of the self), but a deeper isolation (Yalom, 1980). No matter how close we can become to each other, we cannot escape the fact that we each enter the world alone and leave it alone. May and Yalom succinctly capture the point made by Mijuskovic (1979) that

there is a fundamental loneliness; the individual cannot escape the knowledge that (1) he or she constitutes others and (2) he or she can never fully share his consciousness with others. (May \& Yalom, 1989: 378)

An inner conflict then exists between our awareness of this isolation and our wish to connect with others, to be protected and to be part of something greater than ourselves (May \& Yalom, 1989). According 
to Heidegger, the desire for the security of being part of a group can motivate some to forego their individuality (Heidegger, 1962). Yet humans also find meaning for their lives through relationships (Buber, 1958).

\section{(3) Meaninglessness}

Meaning is a common thread throughout existential topics, as existential theory assumes that we always seek meaning in order to make our existence bearable (Becker, 1973). The question is whether we seek meaning (i.e. there is meaning in the world which we aim to discover) or whether we create it (i.e. our existence is devoid of meaning except the meaning we create). Thus, our inner conflict derives from being creatures who seek meaning thrown into a world apparently devoid of meaning (Yalom, 1980).

Jean-Paul Sartre maintained that "existence precedes essence" that is, humans exist before they have a meaning in life. According to Sartre, there is no God to envisage a purpose for us before He brings us into existence, so we come into existence first and create a meaning for our own lives later (Sartre, 2007 [1947]). Viktor Frankl argued that meaning cannot be invented but must be discovered. He believed that meaning is so crucial that he based his "logotherapy" on the principles that life, no matter how wretched, always has meaning; our main motivation to live is to find this meaning; and we always have the freedom to discover meaning. According to Frankl, we can find meaning in life in three different ways:

1. by creating a work or doing a deed;

2. by experiencing something or encountering someone; and

3. by the attitude we take toward unavoidable suffering. (Frankl, 1959: 176)

\section{(4) Freedom}

Although freedom is usually seen in a positive light, in existentialism it is something to which one is "condemned" (Sartre, 1956). The individual is the architect of her own life, choices and behaviour, and with this freedom comes responsibility. For Jaspers, Kierkegaard, Nietzsche and Sartre, freedom means a complete freedom to define the self. Yet if it is true that we define ourselves and our world, then there is nothing to anchor or ground us - there is only an abyss or a void (May \& Yalom, 1989). Our inner conflict arises from our awareness of this freedom on the one hand and a wish for something to ground us on the other hand (May \& Yalom, 1989).

Karl Jaspers (1971), however, believes our freedom is limited by "boundary situations", which include death, suffering, guilt, chance and conflict. Otto Rank (1989) believes that the more an individual is 
unaware of the factors which limit his freedom, the more he is controlled by them. Yet, as Frankl (1959) found, even in circumstances when one's freedom is cut down to the minimum, such as it is in a concentration camp, one still has the most basic freedom - to decide the attitude one takes towards one's fate.

With freedom comes the responsibility that is then associated with the choices one has freely made. As Sartre (2007 [1947]) states, we are our choices, and must accept the responsibility that accompanies them. Encapsulated in this statement is the notion that the self ultimately consists of what we have control over. As Tillich states, "[m]an's particular nature is his power to create himself" (1960: 11). R. D. Laing, who is said to have been influenced by Sartre's existential theories, believed that society undermines individual freedom, and that people who have been diagnosed as mentally ill are simply victims of a "double-bind" in which society has placed them. These are situations in which the individual is left with an impossible choice and something becomes compromised - usually one's psychological stability (Laing \& Esterson, 1970). Laing also believed that this double-bind occurs in families which place conflicting demands on their children. In Sanity, Madness and the Family, Laing and Esterson (1970) provide case studies which show how lies become perpetuated within families, making it difficult for a child to break out of their "bind" and reach the truth of their situation.

Cooper, Esterson, Foucault, Laing and Szasz led the antipsychiatry movement, rejecting the medical model of psychiatric disorder and arguing instead that they are simply labels placed on individuals who do not conform to societal norms and views of reality (Graham, 1986). In The Divided Self (1959), Laing proposes that psychiatric illnesses all have a psychological origin, and that medical treatments interfere with the natural course of the condition, which should cure itself. Here, Laing also attempts to get "inside the mind" of a schizophrenic and finds that if one listens carefully, a schizophrenic person's thoughts and behaviours are understandable, but they have an extreme insecurity which makes mundane circumstances seem threatening. In Self and Others (Laing, 1961), Laing further suggests that schizophrenia should be understood not as something which takes place inside one's head, but as a process which takes place between people, called the family interaction model.

Although Laing and the antipsychiatry movement provide an interesting application of existentialism to the practice of psychiatry, my analysis does not - and cannot - extend to lend support or otherwise 
to the larger claims made by antipsychiatry. Rather than attempting to ascertain the causes (psychological or otherwise) of depression, to question the psychiatric categories (although several interviewees certainly did) or to propose a new understanding of these categories, the analysis presented in this book of how experiences of depression may illuminate perceptions of the self reveals a striking resemblance to the Sartrean branch of existentialist philosophy which emphasises the importance of being aware of, and embracing, one's freedom to choose how one is to be. Nevertheless, Laing's approach has some relevance to this investigation in that the potential influence of social and family dynamics on the development of mental illness flags them as potential triggers for depression, of the sort discussed in Chapter 5. More than any other existential "given", those I interviewed repeatedly returned to the "given" of freedom and control in all aspects of the self explored throughout this book. Specifically, it is Sartre's emphasis on the optimism brought about by an awareness of one's freedom which implicitly arose within the interviews. By revealing how pivotal a sense of control is to views of the self - particularly to how one views one's future self - those I interviewed lend support to this aspect of Sartre's existentialism.

In contrast to other views such as the Socratic, with its emphasis on self-examination and understanding oneself (Nehamas, 1999), or the psychological view, in which authenticity means to live according to the values of one's "inner being" rather than one's society or upbringing (Wood et al., 2008), interviewees viewed themselves as most "authentic" when they felt a greater degree of self-determination (or freedom). Ledermann, whose book explores existential therapy, expresses the notion thus, "[t]he person striving to achieve a greater freedom is also aiming at a greater degree of authenticity" (Ledermann, 1984: 3). The necessity of attaining a sense of freedom to attaining a sense of authenticity will become apparent in Chapter 4.

\section{Situating the Book within Studies of the Self}

Charles Taylor (1989: 34) asserts that " [w] hat I am as a self, my identity, is essentially defined by the way things have significance for me ... To ask what a person is, in abstraction from his or her self-interpretations, is to ask a fundamentally misguided question, one to which there couldn't in principle be an answer." It is for this reason that the phenomenology of people's experiences of depression, its significance for them and their selfinterpretations can progress our understanding of the self. In pursuing 
this path, one must bear in mind that "the way things have significance" for the individual is necessarily informed by the gender and culture which the individual occupies. For instance, Carol Gilligan (1982) found that women define the self much more in terms of their relationships (mother, daughter, wife, etc.) than do men, who define themselves more in terms of independence and individuality than in terms of connection. ${ }^{5}$

Taylor (1989) highlights how the modern concept of the self is always rooted in terms of what is morally valued as good. This, in turn, differs from historical concepts of the self because what was valued was different, and there existed different narrative forms and different understandings of social ties. He also contends that, similarly, notions of "the good", narrative forms and understandings of social ties which are present in different cultures translate into different concepts of the self. As what is deemed valuable by the community is entwined with what is of significance to the individual, Taylor contends that culturally and historically embedded notions of morality are necessarily entwined with concepts of the self. Different concepts of the self are indeed evident across cultures (Cousins, 1989; Geertz, 1973; Kondo, 1990; Lienhardt, 1985; Markus \& Kitayama, 1991; Triandis et al., 1993). Indeed, Geertz (1979) argues that the concept of the self is specific to the Western world. Any study of the self which incorporates people from different genders and cultural backgrounds would then need to take such factors into consideration to account for differences in notions of the self. Interviewing middle-class women in a Western society thus minimises the role that gender and cultural differences would have in explaining the different notions of the self which are presented.

Ascertaining how an illness affects the self helps us to learn more about the self. Sociological investigations of the self revolve around notions of congruence, coherence, consistency and discrepancy (Rogge, 2011), and much mental health research assumes self-consistency and self-congruence to be essential to the self (Grawe, 2004). While self-congruence has been the focus of many relatively recent sociological studies of the self in mental health (e.g. Ball \& Orford, 2002; Burke, 1991; Kaufman \& Johnson, 2004; Paul \& Moser, 2006), other sociological studies have focused on the meanings of life events to individuals (e.g. Charmaz, 1983; Ezzy, 2001; Francis, 1997; Reynolds \& Turner, 2008) as the ways in which individuals make sense of their social reality interconnects with their notions of self. It is with the latter focus that Chapter 5 is concerned, although, as the reader will see, I take a slightly different approach, focusing on the individual's perceptions and attributions of meaning. Rogge believes that, especially when teamed with qualitative methods, such an 
approach is "capable of providing a detailed account of an individual's concerns, meanings and life worlds. The neglect of inter-individual variance, for example as seen in life event research in positive psychology, can be countered by this approach" (Rogge, 2011: 61).

Initial sociological studies of chronic illness centred on Parson's (1951) sick role theory and labelling deviance (e.g. Gerhardt, 1989). More recently, approaches to this area have built on the work of Glaser and Strauss from their grounded theory perspective (Glaser \& Strauss, 1967) with the aim of elucidating the meaning of the chronic illness experience to the individual. One influential idea within this literature is Bury's (1982) concept of chronic illness as a "biographical disruption". The literature has since developed this notion of disrupted biographies. Strauss and Corbin (1987) divide it into three aspects: biographical time, conception of self and bodily capacities. This book is concerned with the second of these aspects.

As several authors have found, chronic illnesses and acquired disabilities impact on and challenge the self, especially as they occur after an adult identity has been established (Bleuler, 1950; Brooks \& Matson, 1987; Bury, 1982; Charmaz, 1991; Corbin \& Strauss, 1987; Fine \& Asch, 1988; Freud, 1958; Kraepelin, 1904; Schneider \& Conrad, 1983; Sullivan, 1940). Such illnesses force the individual to come to terms with this challenge in a society which regards deviances from the norm as indications of both moral and physical inferiority (Weitz, 2001).

As Hydén (1997) notes, chronic illness changes the relationship of the individual with her body, the world and her self. The disruption of the individual's life also means a disruption of the individual's identity (Bury, 1982), as individuals are forced to adjust their life narratives and identities in relation to the illness (Hydén, 1997). Toombs (1988: 207) expresses the idea well when he writes that "[i]llness is experienced by the patient not so much as a specific breakdown in the mechanical functioning of the biological body, but more fundamentally as disintegration of his 'world'". This is evidenced by the way in which interviewees tell their story, for, in both my study and Hydén's, individuals do not talk so much about their symptoms before and after treatment but rather about what happened to their "self" before and after (Hydén, 1995). This idea is also demonstrated in the literature on narratives, in which recovery is presented as realising the authentic self (Maslow, 1976; Stevenson \& Knudsen, 2008), restoring or reconstructing the self (Kohut, 1977; Williams, 1984), regenerating the self (Hawkins, 1990), creating a new self (Ridge, 2008; Schafer, 1992) or discovering a newborn self (Hawkins, 1993).

The notion of "stories" and "narratives" becomes useful here as a way of portraying the meanings present in these transformations (McAdams, 
2001). Bruner (1986), McAdams (2001) and Polkinghorne (1988) suggest that the issues under discussion here are best understood through the "language of narrative". This is because, as Widdershoven (1993) contends, a life and its story are inseparable, as its meaning resides within the story. Life stories examine a life, or part of it, through the eyes of the individual, and are essentially narratives as they are underpinned by narrative discourse (Bertaux, 1981). However, life stories are usually recounted in a storytelling format, giving their narratives the "flavour of fiction, or of fictional accounts of what happened in a person's life" (Denzin, 1989: 42). They also refer implicitly to "the totality of a person's experience" (Bertaux \& Kohli, 2009: 43).

Riessman (1993) emphasises the connection between life stories and identity, as individuals essentially become their autobiographical narratives. Giddens also states that "[a] person's identity is not to be found in behaviour, nor - important though this is - in the reactions of others, but in the capacity to keep a particular narrative going" (1991: 54). In contrast, others such as Strawson (2004) believe that identity need not take a narrative structure. While the "language of narrative" will at times be useful in this book, I do not wish to say that identity, or the experiences recounted by the interviewees, is only to be understood through this language, as experience can occur in different registers.

Research into life stories shares a connection with autobiographical memory, as the latter also uses a life story to help define the self (McAdams, 2001). Although this book elicits memories from interviewees as they recount their "illness narratives", there are some important differences between it and studies in autobiographical memory. First, although an investigation into the individual's account of how her depression fits into her past, present and future self involves uncovering memories which are key to her account, I do not presuppose that memories must be associated with meaningful aspects of the self and depression, whereas studies in autobiographical memory do not allow for the absence of an associated memory as a meaningful possibility. Second, autobiographical memory constitutes a wide range of personal experiences and information, whereas life stories are more limited in their scope, made up of a series of "temporally and thematically organized scenes and scripts that together constitute identity" (McAdams, 2001: 117). This point of difference is even further accentuated in the following chapters, which work within an even more delimited set of "scenes and scripts" known as "self stories".

Self stories, in contrast to life stories, focus on the self in relation to a particular experience. Although they, too, are personal narratives, they are recounted by the individual in relation to a certain type of experience (Denzin, 1989). Denzin, for example, examined how alcoholics represent 
themselves to each other through their self stories in Alcoholics Anonymous meetings (Denzin, 1989). In seeking women's stories of their depression and recovery and their representations of their self in relation to depression, I elicit self stories rather than life stories. In doing so, I not only focus on the self specifically in relation to depression rather than their life as a whole but also embrace the "multiplicity, variability, and context specificity" of the small story level which allows for the multiple identities that can emerge in a conversational context (Bell, 2009: 282). As Georgakopoulou writes, big stories represent a "longstanding privileging of ... a unified, coherent, autonomous, reflected up and rehearsed self" (2006: 128), whereas small stories are a new trend in narrative " $\ldots$ that allows for, indeed sees the need for a scrutiny of fleeting, contingent, fragmented and multiple selves" (2006: 128). Here, the image of the "bricoleur" (Lévi-Strauss, 1966) becomes useful, as the self within these self stories is constructed from the available material or "story lines" at hand in society (Hydén, 1995), such as those within the media, literature, friends, family and practitioners. As in self stories, the self in the "bricoleur" metaphor is "ineluctably local" (Geertz, 1983), as our biographical work consists of pulling together facets of our personal history for our current purposes (Gubrium \& Holstein, 1995; Gubrium \& Holstein, 1998; Gubrium et al., 1994).

This book's slant towards a small story approach is driven by my desire to avoid the temptation to mould the data into a coherent narrative and instead to allow for the possibility of insight that can be gained from the shifting and sometimes contradictory positions that individuals can take. See, for example, Singh's (2005) study of parents of children diagnosed with Attention Deficit Hyperactivity Disorder (ADHD) which, in presenting the contradictions in parents' dosing decisions, reveals their underlying ideas concerning authenticity. The small story approach is thus well suited to examining the self through the lens of depression.

Within qualitative studies of health and illness, the "work-of-living" with health and disease has been categorised into five phases by Jensen and Allen (1994): (1) recognising the threat; (2) defending and protecting the self; (3) reconciling the change; (4) learning to live again; and (5) living again. Alternatively, Morse and Johnson (1991) have categorised the trajectory into four phases: (1) uncertainty; (2) disruption; (3) striving to regain the self; and (4) regaining wellness. Within studies of the impact of chronic illness on the self, the majority of the literature is split between describing it either as a biographical disruption (Bury, 1982), a loss of the self (Brody, 1994; Charmaz, 1983), a deconstruction/reconstruction of the self (or narrative reconstruction) (Davidson \& Strauss, 1992; Hydén, 1995; Sells 
et al., 2004; Williams, 1984; Williams, 1997), or as the self becoming redefined or reshaped (Romano et al., 2010; Wisdom et al., 2008).

Although I draw on this literature to inform and contrast with my analysis of the interview material, particularly in Chapter 2, I refrain from framing the significance of depression to the self entirely within the frameworks described above as such frameworks risk imposing certain assumptions onto the material. For instance, is depression necessarily experienced as a biographical disruption? As shown later, this is not always the case. By the same token, narrative reconstruction, which, as Williams (1997: 209) describes, is "an attempt to reconstitute and repair ruptures between body, self, and the world by linking-up and interpreting different aspects of biography in order to realign present and past and self with society" was not undertaken, or seen as necessary, by some of the women I interviewed as they did not consider that there was anything to "repair".

Chapter 2 focuses more specifically on the ways in which depression is described in relation to the self. Given this focus, the themes identified in the chapter differ slightly to the themes identified in the literature on the self in chronic illness more generally. Sometimes, the difference in themes is more of a difference in name than in thematic content; for instance, the following quotation is presented by Wisdom et al. as an example of the "loss of self" theme:

[Bipolar disorder] is a disease that for me, literally steals me from myself - a disease that executes me and then forces me to stand and look down at my corpse. It is what the criminal lawyer in me calls a medical examiner's antithesis: life by strangulation (Hartmann, 2002). (As quoted in Wisdom et al., 2008: 491)

The quote could also be considered an example of the "enemy of the self" or "illness" themes presented in Chapter 2. The chapter draws on some of the themes present in the literature on the self in depression rather than in the literature on chronic illness more generally, for three reasons: (1) the former themes have been developed specifically in relation to depression narratives; (2) not all interviewees were of the view that their depression is a chronic illness, which challenges the degree to which themes in narratives of the self and chronic illness are applicable to depression; and (3) the themes within the literature on the self in depression align more closely with the terminology that interviewees used to describe their depression. 


\section{Defining Authenticity}

Just as there are many ways to define the self, so it is with authenticity. I will here present a few contrasting definitions of authenticity (which are by no means exhaustive of the variety) before presenting the definition I will be working with in this book. This book is not concerned with what comprises authenticity in a metaphysical sense. Rather, it is concerned with authenticity in the existential sense - that is, the sense in which it is invoked in discussions of responsibility and moral psychology. That is, what it is to think, feel and act in a way that expresses what one truly is. As Bernard Williams explains, it is "the idea that some things are in some sense really you, or express what you are, and others aren't" (Guignon, 2004: viii). Another connotation, according to Monica Betzler, is "being oneself". As Betzler (2009) notes, if the term is not to be interpreted superficially, it must mean that it is possible to not be oneself in the sense of betraying or failing oneself.

While these descriptions allude to what it is to be true to oneself, several scholars have also linked authenticity closely with autonomy such that one of the conditions of autonomy is authenticity (e.g. Betzler, 2009; Dworkin, 1976; Meyers, 1989; Ryan \& Deci, 1999). For instance, Carver and Scheier (2000: 285) describe how part of what constitutes autonomy is not only to be free from external impediments but also to act authentically, according to what one truly values. They write:

In an early draft of our commentary, one of us wrote that self-determination can be exercised by stepping onto a busy highway without looking, but that's not right. Internal perceived locus of causality could (we assume) be reflected in such an act, if the impetus to act originates inside the person's mind. So could self-governance, in the sense that the decision to act is made on one's own with no outside interference (the dictionary definition of autonomy). The act could be freely chosen. But upon further review, such an act probably would not be autonomous in the Deci and Ryan view, because it fails to advance a value of the true self, and indeed may conflict with an important value of the true self (desire for self-preservation).

One problem with definitions of autonomy and authenticity such as these is that they are conceived as asocial, giving the impression that the true self is either disconnected from, or in opposition to, outside agendas. This view of authenticity is in stark contrast to Taylor's, in which authenticity is not just an individualistic struggle but one which must involve a connection with one's social and moral context and with projects larger than oneself in order to derive meaning (Taylor, 1991a). In other words, external influences can be viewed as part of the dynamic and mutually influencing relationship between a person and her environment. The 
human mind, brain and behaviour are socially embedded and it is difficult, if not impossible, to separate them from the social context in which they operate.

Another problem with determining authenticity is that it may be difficult to distinguish one's authentic self from one's ideal self (if an authentic self even exists). That is, the desire to view oneself as authentic in certain mental/ emotional/behavioural states and not others may be motivated by the desire to present one's ideal self or best self as authentic, which may or may not coincide with one's true self. Relatedly, Loe and Cuttino (2008: 309) write:

If an individual's goal is always "authenticity," the phenomenological emotional experience of feeling true to oneself (Taylor, 1992; Vannini, 2006), then medicine can create a sense of inner conflict as the gap widens between perceived "authentic" and "ideal" identities.

Chapter 4 not only provides support that this sense of inner conflict indeed exists among some individuals but also goes some way towards unpacking why it exists - that medication's perceived challenge to selfdetermination lies at the heart of this conflict.

In the chapters that follow, my empirical investigation explores what the phenomenology of authenticity and the self in different states (depressed, medicated and so on) might tell us about these concepts. The potential for the phenomenology of these concepts to further our knowledge of them is particularly salient in the case of authenticity because what it is to be authentic is often understood to be intimately tied with what it is to feel authentic. In fact, as mentioned above, several authors regard authenticity as an affective state of feeling true to oneself (e.g. Erickson, 1995; Gordon, 1989; Harter, 2005; Salmela, 2005; Schwalbe, 1993; Turner \& Schutte, 1981). Asking individuals about the states in which they feel they are their "real self" and why they feel that way reveals a common thread within what authenticity means to them. If we take authenticity to be intimately tied to feeling true to oneself, then these accounts support certain facets of the way authenticity has been defined thus far while challenging others.

The concept of authenticity I support is one which allows for the influence of society and morality, and places autonomy (or self-determination) front and centre, such that the state in which the individual possesses autonomy determines the state in which one is authentic. ${ }^{6}$ (In fact, according to Trilling (1971: 122), one connotation of the Greek for authenticity (authentheo) is "being in control".) Such a definition implies that an action can be considered my own and authentic even if it is not consistent with my best intentions, as long as I retain control of my actions. Such an account of authenticity also allows us to say that one need not be reflective in order to 
be authentic. As long as the individual is in control of her actions, she can be considered to express her authentic self, regardless of whether she reflects on her self-state or not, and regardless of whether her actions are in line with her ideal, or even best, self. This is the definition of authenticity I favour and which is suggested by, and consistent with, the empirical evidence in this book. Chapter 4 provides support for, and suggests an added nuance to, the relationship between autonomy and authenticity.

\section{Defining Control}

The interviews contained in this book advance our knowledge of the self and authenticity by providing empirical support for particular aspects or interpretations of these concepts. The individuals I spoke with made it apparent that their sense of self depends upon their sense of control. In other words, individuals indicated that differences in how they view their self depend on differences in their sense of control. A sense of control was invoked in different ways depending on the context. For instance, when speaking of authenticity, the sense of control that the individual invoked was self-determination. When speaking of one's future self, individuals invoked a sense of control over future episodes of depression as being significant. As such, it does not make sense to provide a hard definition of control here as I have done for the self and authenticity in order to show how the empirical evidence corroborates or contrasts with such a definition. Instead, I will clarify the different senses in which "control" is invoked by the interviewees in order for the reader to understand the nature of this crucial lever.

It was clear from the context of the interviews and my follow-up questions that those I interviewed used "control" in slightly different ways depending on the subject of discussion. In Chapter 2, there were those who described themselves as being an "out of control self" when depressed or manic, and those who felt that depression was out of their control. Some professed to feeling both ways at different times. In either case, their sense of control was used to explain their view of depression. First, "control" is used in a similar sense to "self-control", meaning an ability to control their thoughts, emotions and behaviours such that they would not become depressive thoughts, emotions and behaviours. Berkowitz's (1982: 225) definition of self-control as "the ability to intentionally manipulate covert mental events, most notably inner speech and images, in order to regulate one's own behaviour" is the most relevant definition here and is common to many other understandings of self-control. "Control over depression" was also invoked by the individual as an ability to change her environment or to take action such that she would not become depressed. Chapter 5 also 
invokes both these senses of control - either the ability to control one's thoughts, emotions and behaviours such that they would not become depressive thoughts, emotions and behaviours; or the ability to change one's environment such that one would not become depressed.

Closely linked to both these senses of "control" is "self-determination" or "autonomy". Chapters 3 and 4 rely on this nuance of the concept more so than the other chapters. The more the women felt they could determine aspects of their self (i.e. their choices, actions, behaviours and so on), the more they felt authentic. Conversely, the more they felt that another force (e.g. a biochemical imbalance or an antidepressant) was determining these aspects of their self, the less control they felt they had over them. This is congruent with the dictionary definition of autonomy being the ability to act, choose, etc. without external impediments. Autonomy has also become closely intertwined with what it means to be authentic, so what these women say about authenticity also has implications for its relationship with autonomy.

\section{Overview}

A typical journey through depression moves from its first appearance, to diagnosis, to treatment (medical or otherwise) and finally to recovery or stabilisation. The journey may not be linear and may go back and forth or skip a step or two, but the women I spoke to had been diagnosed with depression, had undergone treatment for it and felt better. They were therefore in a position to provide insight into the impact of each stage of depression and recovery on their self. What emerges is a relationship between perceived control and the self. Each chapter elucidates the contours of this relationship as it arises within each stage of depression and recovery.

Chapter 2 presents the different ways that depression is viewed by the individuals I spoke with and is in keeping, as much as possible, with the ways in which it is described by the individuals themselves. It also examines the contexts in which those views are presented, the ways they are frequently combined and the reasons why individuals choose one theme over another or a combination of themes. Chapter 3 focuses on how individuals first react to their diagnosis and what impact it has on their sense of self. Prior explorations of how individuals react to being diagnosed with depression have documented shock, fear, relief and denial. While these reactions were also voiced by the women I spoke to, my exploration of why they reacted in the way that they did shows that their reactions were premised on a biomedical understanding of depression at the point of diagnosis. 
In Chapter 4, I examine how individuals view the role of medication in relation to their responsibility for recovery, as well as their reflections on the notion of an authentic self, what they believe to be essential for authenticity and how/whether psychiatric medication presents a challenge to it. In meta-syntheses of the literature in this area conducted by Malpass et al. (2009) and Khan et al. (2007), control has been recognised as an important issue in treatment for depression, influencing how individuals feel about their medication as well as their self on medication. I shall also propose that perceived control (qua self-determination) plays a pivotal role in individuals' views of authenticity, adding detail to the part it is understood to play within individuals' perceptions of the self in depression.

Chapter 5 demonstrates that an individual's beliefs about how depression began can be significant for her views of depression and its role in her life - particularly its role in her future self. I shall show that those who considered most of their episodes to have been triggered by events or circumstances in their lives were more likely to believe that their depression could in future be overcome, whereas those who did not consider most of their episodes to have been triggered were more likely to believe their depression to be chronic. The final chapter will then present the implications of the previous chapters for our understanding of the self and the way depression is treated. 